\title{
Actualités chez nos confrères français
}

\author{
Jean Martin \\ Dr méd., membre de la rédaction
}

L'Ordre national des médecins français publie un bulletin bimestriel, médecins, que je reçois de longue date. Le premier numéro de 2018 [1] traite plusieurs thèmes d'intérêt. Innovation en médecine - Une vision du futur. C'est le titre d'un dossier à propos des évolutions rapides voire fulgurantes des technologies et des moyens diagnostiques et thérapeutiques. Un exemple: en chirurgie ambulatoire, l'Assistance publique déploie en région parisienne un outil de suivi par SMS des patients à domicile. «L'intelligence artificielle et un algorithme de questions et réponses permettent à un robot conversationnel de dialoguer avec les patients avant et après leur opération. «Avez-vous une douleur non calmée par les médicaments? Si oui, évaluez-la sur une échelle de 0 à 10;; ‘Avez-vous l'impression que le pansement est trop serré?`; ‘Si tout va bien, répondez TVB.»» Réduction du nombre d'appels aux patients par les équipes soignantes, gain de temps, moins de retards et d'annulations. Et le patient est responsabilisé. A l'horizon 2020, dit-on, deux tiers des actes chirurgicaux pourraient se faire en ambulatoire.

Il n'est pas question de s'opposer aux développements technologiques, mais certaines mises en garde sont judicieuses: «La puissance de l'intelligence artificielle ne doit pas faire fi des enjeux éthiques [...] Aussi séduisantes soient-elles, ces promesses ne doivent pas faire oublier les questions en suspens [...] Utilisation et anonymisation des données pour la recherche: un équilibre à trouver.»

Formation médicale. L'Ordre et la Conférence des doyens de médecine veulent la faire évoluer. Parmi les propositions faites: 1) promouvoir la découverte des hôpitaux non universitaires; 2) encourager les enseignements pluri-professionnels; 3) stages de découverte de la médecine générale; 4) élargir les origines sociales et géographiques des étudiants; 5) sensibiliser à la responsabilité collective et sociale.

Déontologie. Est évoquée la réunion d'octobre 2017, à Chicago, de l'Association médicale mondiale (AMM), avec une révision de la Déclaration de Genève (version moderne du serment d'Hippocrate). Pour la première fois, le serment fait référence au respect de l'autonomie du patient (en 2017... il aura fallu du temps). Est intro- duite aussi l'obligation pour les médecins de partager leurs connaissances médicales au bénéfice des patients. Violences faites aux enfants. Entretien croisé entre quatre femmes médecins. "Chaque jour, plus de deux enfants décèdent de violences physiques en France.» Propos d'une psychotraumatologue: «Les violences subies pendant l'enfance représentent le premier risque de suicide, de dépression, de conduites à risque, mais aussi de troubles cardio-vasculaires et d'autres problèmes.» D’une pédiatre: "Chez le médecin traitant, une forme de déni peut apparaître, pour ne pas être confronté à l'impensable [...] En amont, il est essentiel de mettre plus de moyens dans la prévention auprès des familles présentant des fragilités (chômage, séparation, etc.). Et de repérer plus précocement [...] Tous les professionnels concernés doivent bénéficier d'un référentiel commun d'évaluation.» D'une pédiatre légiste: «Il faut créer des pôles interdisciplinaires dans les hôpitaux pour prendre en charge globalement ce problème de santé publique majeur.»

Climat. L'assemblée susmentionnée de l'AMM a appelé les gouvernements et les acteurs non étatiques à reconnaître les graves conséquences sanitaires du changement climatique, en demandant un financement adéquat de la lutte contre ce changement. Sur le même sujet, l'Ordre national a organisé un débat dans le cadre du «One Planet Summit», en décembre dernier à Paris. Y a été présentée la dernière version du rapport de The Lancet, «Compte à rebours santé et changement climatique» [2]. Conclusions dudit débat: «Nul ne peut désormais ignorer les effets du climat sur la santé. Les changements climatiques ont et auront un impact majeur. Les professionnels de santé doivent aujourd'hui faire entendre leur voix.»

Un signe. Le président de l'Ordre vient d'être nommé membre correspondant de l'Académie nationale de médecine. Il est le premier médecin généraliste à y entrer.

\section{Références}

1 Médecins. Bulletin de l'Ordre national des médecins, 4, rue Léon Jost, F-75855 Paris Cedex 17, n ${ }^{\circ}$ 53, janvier-février 2018.

2 "Countdown on health and climate change: from 25 years of inaction to a global transformation for public health.» Une citation: "Anthropogenic climate change threatens to undermine the past 50 years of gains in public health.» 